\title{
New directions in modelling, analysis and design of WDM/OFDM-networks: (I) Optical Switching
}

\author{
Josef Giglmayr \\ Heinrich-Hertz-Institut für Nachrichtentechnik Berlin GmbH \\ Einsteinufer 37, D-10587 Berlin \\ E-mail: Giglmayr@hhi.de
}

\begin{abstract}
Bearing in mind that a topological representation of a finite graph is a compact space, by the relationships between (Graph)Topology, Algebraic Topology, Group Theory, (Differential)Geometry and Combinatorics a new universe arises for modelling, analysis and design of (telecommunication) networks. Throughout the present paper, coverings/covering spaces, the lifting problem and quotient graphs are briefly interpreted in terms of the requirements for the combinatorial design of optical switches though the presentation is mainly based on illustrations rather than on a (precise) mathematical description.
\end{abstract}

Key words: Multi-layer, parallel waveguides, electrodes, spatial all-optical switch, planar alloptical switch, all-optical expander/concentrator, parallel permuters, vertical stacking, graph covering, lifting

\section{Introduction}

Graph models are fundamental tools in modelling, analysis and design of communication networks. Mathematicians have recognized the limitations of ordinary graph models and developed several generalizations such as pseudographs and hypergraphs, respectively. Pseudographs are multigraphs ( $\equiv$ multiple edges between some nodes) with (multiple) self-loops. For example, the de Bruijn directed graph (digraph) $B(\Delta, D)$ with $D$ edges between every node-pair and self-loops at some nodes provides the nonblocking simultaneous communication of the node-pairs [1]. In contrast, hypergraphs are graphs with more than two nodes for every edge [2]. Pseudographs may be applied to model WDM/OFDM-networks consisting of optical fibers interconnecting optical nodes and access nodes (physical layer), respectively. By the partition of access nodes into intersecting subnets a hypergraph is formed (logical layer) [3].

Throughout the paper, a topological approach is applied where finite simple regular graphs (and even infinite, nonregular, multigraphs, pseudographs or hypergraphs) are the most simplest space [4]. The next more complicated topological spaces are (compact) surfaces which are of interest once pseudographs are embedded into them. One basic problem of topology is to determine whether there exits a continuos function (and its inverse) between two given topological spaces. Therefore, topology is, roughly speaking, called the geometry of continuous functions. Here the concept of covering projections/covering spaces and of quotient maps/quotient spaces comes in. Throughout an application of topology to networks, these geometric problems have to be treated by means of powerful algebraic methods. The aim of the ongoing work is to deduce some valuable hints for the modelling, analysis and design of WDM/OFDM-networks and their components.

Throughout the paper the application of graph coverings is shown for the design of all-optical switches, expanders and concentrators. However, the most convenient treatment of graph coverings is by voltage graphs [5] which will not be shown in this paper. Another problem are liftings which are briefly mentioned throughout the discussion of switches, expanders and concentrators. 


\section{Preliminaries}

In the following minor elements from topology [4] and graph theory [5] are collected which are needed to understand the ideas of the paper.

\subsection{Graph topology}

From the point of view of topology, a graph/network is a rather simple topological space even it may be an infinite, nonregular pseudograph. Note, a topological space $(X, \mathcal{A})$ is a topology $\mathcal{A}$ on a set $X$ where $\mathcal{A}$ represents a family of subsets of $X$ which satisfies some simple conditions [4].

\section{Coverings}

A family $\mathcal{A}$ of subsets $A_{i}$ of $X$ is said to cover $X$ if and only if (iff) $\cup A_{i}=X$. In terms of graph theory, a graph $G$ is a covering of a graph $H$ iff there exist a map $p: V(G) \longrightarrow V(H)$ which induces a one-to-one correspondence between the nodes adjacent to $v \varepsilon G$ and the nodes adjacent to $p(v) \varepsilon H$. Then the graph $G$ is called the derived graph [5] or the covering space [4].

Example 1: The distance-transitive graph/antipodal graph $C_{4}^{(2)}$ (Sub-Section 2.2) in Fig. 1 (a) lhs, which equals the 3-cube $Q_{3}$ [6], is a double cover of $K_{4}$ [Fig. 1 (a) rhs], i.e. $K_{4}$ is contained in $Q_{3}$ with respect to both coordinates [7]. Note, $K_{4}$ covers the ring $C_{4}$ which represents the intersection of the embedded 4-gon prism (Sub-Section 3.2).

Example 2: The bipartite graph $K_{3,3}$ and $C_{3}^{(2)}$ (two connected triangles) both have the same degree. Then there exist a graph $C_{6}^{(2)}$ (two connected hexagons) which cover both subgraphs [8].

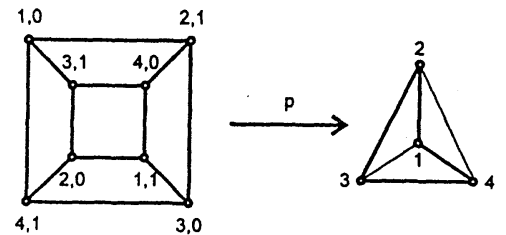

(a)
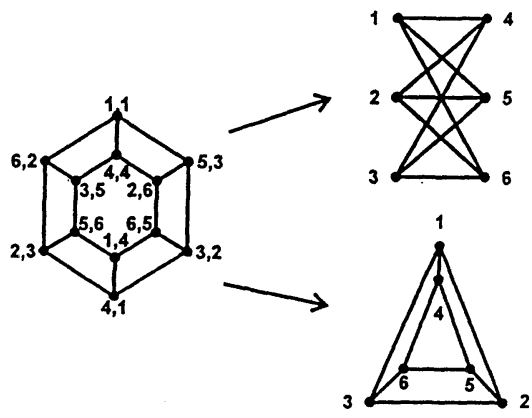

(b)

Fig. 1. Double covers of graphs. (a) The two connected cycles $C_{4}^{(2)}\left(\equiv Q_{3}\right)$ cover the completely connected graph $K_{4}$ twice (Example 1). (b) $C_{6}^{(2)}$ covers the bipartite graph $K_{3,3}$ and $C_{3}^{(2)}$ (Example 2).

\section{Lifting}

The lifting problem in topology is to decide when one can 'lift' a projection/map $f: W \longrightarrow H$ to a map $g: W \longrightarrow G$ where $p: G \longrightarrow H$ is given [Fig. 2 a)]. Similar, the various nonisomorphic coverings are connected by an automorphism which also can be lifted [Fig. 2 (b)]. Liftings may be applied to mappings/projections of topological spaces (graphs/networks or walks/circuits e.g. the boundary of faces $[4,5]$ ) by first embedd the pojected graph $H$ and then lift the embedding such that the covering space $G$ is embedded $[4,5,9]$. 
Remark 1: By the lifting concept, walks/routings in an $N$-gon prism switch (Sub-Section 3.1) may be lifted to walks/routings in an embedded $m N$-gon prism switch (Sub-Section 3.2) and vice versa, walks/routings in the $m N$-gon prism switch may be based on walks/routings in an $N$-gon prism switch. However, presently, the routing of the $m N$-gon prism switch for the rearrangeable nonblocking case is redrawn from an algorithm for cellular arrays $[10,11]$.

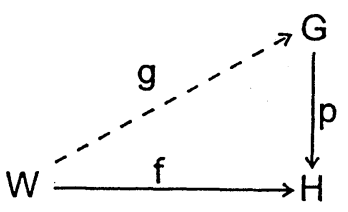

(a)

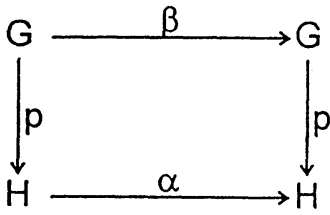

(b)

Fig. 2. (a) Lifting of a mapping $f$ by means of the covering projection $p$ to $g=p^{-1} \circ f$ [4]. (b) Connection of nonisomorphic coverings by a lifted automorphism, i.e. the automorphism $\alpha$ is lifted to the automorphism $\beta$ [12].

\subsection{Graph theory}

The paper deals with distance-transitive graphs, which are the most symmetric graphs, and some definitions and results, which mainly are due to [13], are collected in the following.

\section{Automorphism}

An automorphism of a (simple) graph $G=(V, E)$ (where $V$ is the set of nodes and $E$ the set of edges) is a permutation $\pi$ of $V$ which has the property that $(u, v)$ is an edge of $G$ iff $(\pi(u), \pi(v))$ is an edge of $G$, i.e. it preserves adjacency.

Distance-transitive graph

A graph $G=(V, E)$ is said to be distance-transitive, if, for any nodes $x, y, u, v \varepsilon V$ satisfying $d(u, v)=$ $d(x, y)$ ( $d$ is the distance between 2 nodes in terms of the number of edges) there is an automorphism $\pi$ of $G$ which takes $u$ to $v$ and $x$ to $y$. This is the strongest symmetry condition on graphs which allows to reduce its description by the $N \times N$-adjacency matrix via the description by an $3 \times D+1$ intersection array to a $D+1 \times D+1$-matrix ( $D$ is the diameter) where all the three matrices have the same eigenvalues. Here an interesting eigenvalue problem comes in [14].

\section{Antipodal graph}

An antipodal graph $G$ with diameter $D$ has the property that each node $v \varepsilon G$ has an unique (antipodal) node $\bar{v}$ of distance $D$ from $v$ in $G$. The antipodal node-pairs in $G$ produce a quotient graph $H$ with a double cover projection $p: G \longrightarrow H$ (Sub-Section 2.1). Any antipodal graph $G$ has circuits of length $2 D$ passing through antipodal node-pairs [15]. Here the 'block system' of a graph comes in, which allows to derive a quotient graph whose nodes correspond with the blocks (Note the intersection of the embedded $m N$-gon prism switches in Sub-Section 3.2 are antipodal graphs).

Example 3: The completely connected graph $K_{4}$ in Fig. 1 (a) is a graph quotient of the 3-cube $Q_{3}$ and the double triangle $C_{3}^{(2)}$ in Fig. 1 (b) is the graph quotient of the double hexagon $C_{6}^{(2)}$. Roughly speaking, the $N$-gon is a quotient of the $N+1$-gon. 


\section{Concepts and quantities}

In the following, the elements of the foregoing Section are applied to some novel components of WDM/OFDM-networks. Although there has been obtained a considerable progress in glas fiber research applications, the crucial points are the nodes of the networks which include optical switches and crossconnects ( $\equiv$ a space switch carrying frequency channels), respectively. The space switches presented in the following are aimed to be extended to crossconnects.

\subsection{The $N$-gon prism switch}

For the purpose of the extension of current all-optical switching principles [16] into the 3-D physical space, the $N$-gon prism switch $(N \geq 3)$ has been introduced which is based on multi-layer architectures and nearest-neighbour interconnected $2 \times 2$-switches $[17,18,19]$ (see Fig. 3 for $N=4$ ).

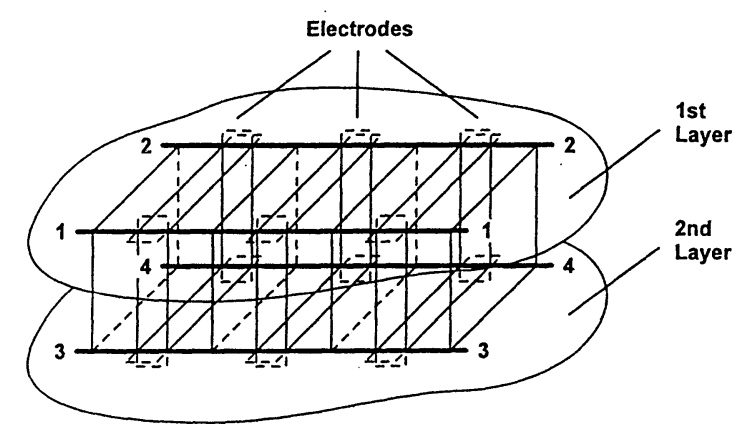

Fig. 3. 3-stage 4-gon prism switch composed of a double-layer, parallel waveguides and electrodes.

The architecture of the $N$-gon prism switch is described for one stage by [20]

$$
C_{N} \oplus K_{2}
$$

where $\oplus$ is the Kronecker sum $(K S)$ [21], $C_{N}$ is a cycle with $N$ nodes and edges and $K_{2}$ represents 2 nodes interconnected by one edge. The number of stages $(N S)$, sufficient for the generation of $N$ ! (arbitrary) permutations ( $\equiv$ rearrangeable nonblocking interconnections) is

$$
N S=N-4
$$

caused by the isomorphism between the $N$-gon and the nearest-neighbour interconnection of switches with skew 1 (Fig. 4) [22].

The number of switches per stage $\left(N S W_{\text {stage }}\right)$ is

$$
N S W_{\text {stage }}=\left\lfloor\frac{N}{2}\right\rfloor
$$

where the 'floor' function $\lfloor a\rfloor$ is the largest integer less than or equal to $a$. From Eqs.(2) and (3) we may conclude that the total number of $2 \times 2$-switches is

$$
N S W_{\text {total }}=\left\lfloor\frac{N}{2}\right\rfloor(N-1)
$$

which is below the Spanke-Benes minimum $N(N-1) / 2$ for $N$ odd. $\operatorname{tant}$

For the proper understanding of the $\mathrm{N}$-gon prism switch the following assumptions are impor- 
(A) Once a $2 \times 2$-switch is formed, no further switch may be established within the same stage with the waveguide involved in this $2 \times 2$-switch and

(B) Spatial diagonal switching is not allowed and is resolved by diagonal switching at the boundary faces/facets of the $N$-gon prism.

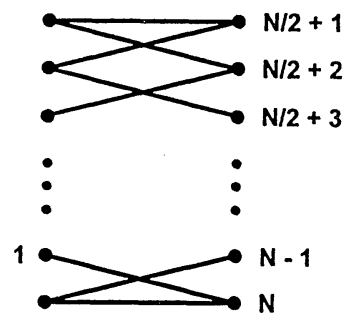

(a)

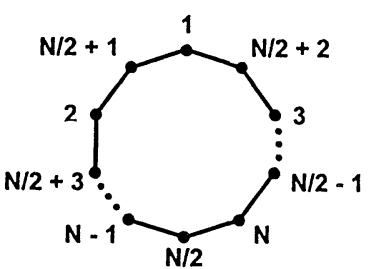

(b)

Fig. 4. Isomorphism between (a) nearest-neighbour interconnections and (b) $N$-gon.

From the switching principles (A) and (B) we may conclude that the moving location of switches $(S W)$, expressed by the number of their possible arrangement $(N P A S W)$ within the $N$ gon prism switch, determines the improvement in Eqs.(2) and (4) compared with the Spanke-Benes network. $N P A S W$ is shown for some $N \geq 3$ in the following Table 1 and Fig. 5 .
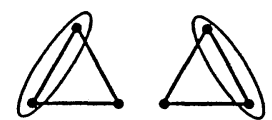

(a)

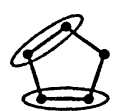

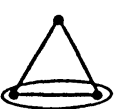

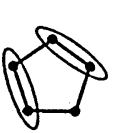

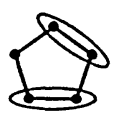

(c)

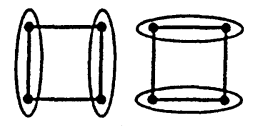

(b)
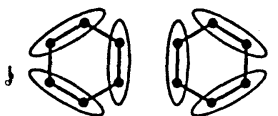

(d)

Fig. 5. Possible arrangements of $2 \times 2$-switches for (a) $N=5$ to (b) $N=6$.

Table 1. Number of possible arrangements of $2 \times 2$-switches at one stage of the $N$-gon prism switch.

$$
\begin{array}{l|cccccccc}
N & 3 & 4 & 5 & 6 & 7 & 8 & 9 & 10 \\
\hline N P A S W & 3 & 2 & 5 & 2 & 2 & 2 & 2 & 2
\end{array}
$$

Example 4: For $N=3$ the Spanke-Benes network has 3 stages and 3 switches which provides $2^{3}=8$ 
states sufficient to generate $3 !=6$ permutations [16]. However, the 3 -gon prism switch contains 2 switches and is thus represented by $2^{2}=4$ states which is not enough to generate 6 permutations. But, according to Table 1 , the number of possible states is $3 \times 4=12$ which now is sufficient.

Remark 2: In Table 1 , the case $N=5$ has the largest $N P A S W$ (Fig. 5), though no advantage seems to arise in Eq.(4).

Remark 3: The routing algorithm of the $N$-gon prism switch is of complexity $O(N)$ and is optimal [11].

\subsection{The embedded $N$-gon prism switch}

Increasing the number of inputs/outputs, the number of layers increases, which, however, may be difficult to set up. Therefore, embedded prism switches are introduced which are expected to be implemented without additional electrodes for switching between the prisms (Fig. 6).

Table 2. Reduction of the average distance $\bar{d}$ by the (connected) embedding of prisms.

\begin{tabular}{l|ccccccc}
$N$ & 6 & 8 & 9 & 10 & 12 & 14 & 16 \\
\hline$N$-gon & 1.50 & 2.28 & 2.50 & 2.77 & 3.27 & 3.76 & 4.26 \\
3-gon & $1.4^{2}$ & & $1.87^{3}$ & & $2.36^{4}$ & & \\
4-gon & & $1.71^{2}$ & & & $2.18^{3}$ & & $2.27^{4}$ \\
\hline
\end{tabular}

$\nu$ means the number of prisms

With the architecture in Fig. 6, for a large number of inputs (e.g. $N=16$ ), we need 8 layers [Fig. 7 (a)]. For the further reduction of the number of layers we may implement the prism switch in Fig. 7 (b) [23] or the 4-gon ${ }^{2}$ prism switch (synonym: $2 \times 4$-gon prism switch) which may be found by (1) line iteration of Fig. 6 (b) or as an embedding into the Kautz digraph $K(2,4)[24]$.

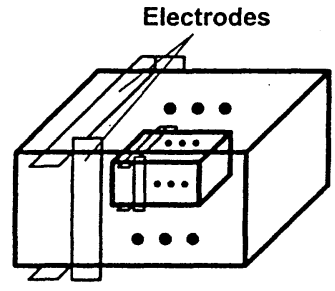

(a)

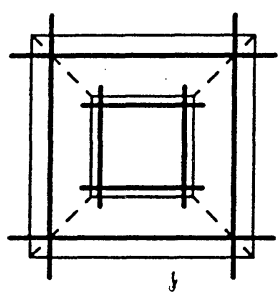

(b)

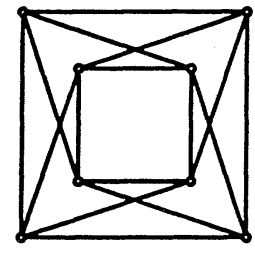

(c)

Fig. 6. (a) Proposed implementation of the embededd 4-gon prism switch. (b) Intersection. (c) Generalization (which might be difficult to implement except in the planar case).

The embedding of one prism reduces the average distance between arbitrary node-pairs (Table 2) and in turn $N S$ for rearrangeable nonblocking interconnections. However, the available routing algorithms show that $N S$ increases considerably for $\nu(=m) \geq 3$ though the obtained results may not represent the minimum [11].

From an analysis of the average distance $\bar{d}$ we may conclude an increase of the average distance $\bar{d}$ and in turn of $N S$ for a decreasing number of layers (Table 3 ). 
Remark 4: The topology of Fig. 7 (a) equals the hypercube $Q_{4}$ except the absence of the interconnections between the outer and inner square in the latter [6]. For $N \geq 5$ and $\nu(=m)=2$ the numbering of the nodes may be according to the dihedral group [25].

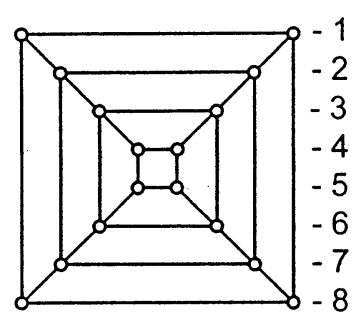

(a)

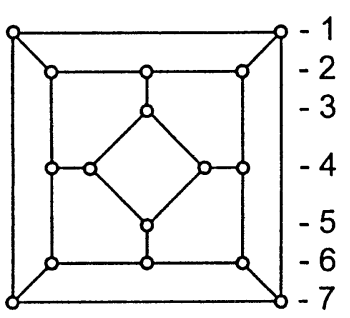

(b)

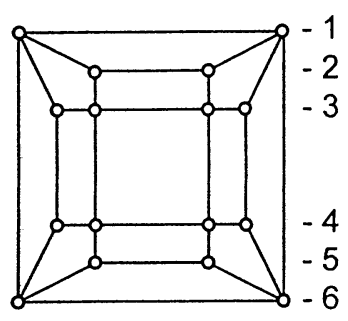

(c)

Fig. 7. Intersection of embedded prism switches. (a) 4-gon ${ }^{4}$ prism switch. (b) Redrawn from [23,Fig. 13]. (c) Redrawn from [24,Fig. 2]. (The digits count the number of layers.)

Table 3. Characterization of distance-transitive graphs (arising by the intersection of embedded prism switches) by the number of layers and by the average distance $\bar{d}$ [the 1st, 2nd and 3rd column is the architecture in Fig. 7 (a),(b) and (c)].

\begin{tabular}{l|ccc} 
No. of Layers & 8 & 7 & 6 \\
\hline $\bar{d}$ & 2.27 & 2.46 & 2.73
\end{tabular}

Example 5: For the 3-gon ${ }^{2}$ prism switch (6 inputs/outputs) $N S=4$ for providing rearrangeable nonblocking interconnections instead of 5 stages for the 6-gon prism switch. For the 4-gon ${ }^{2}$ prism switch (8 inputs/outputs) $N S=5$ instead of 7 stages for the 8-gon prism switch.

Remark 5: The latter $N S=5$ equals the (global) shuffle result $2 \log 8-1$ (lower bound) which always arises iff the intersection graph is a hypercube and which is, after our knowledge, the minimum $N S$ for (local) networks of nearest-neighbour interçnnected $2 \times 2$-switches.

Remark 6: The development of a routing algorithm for the embedding of $m$ - 1 prism switches $(m \geq 2)$ is ongoing work. For example, for two 4 -gon prisms ( 8 inputs/outputs in Fig. 6) a branchand-bound-based algorithm is under development [11].

\subsection{Planar $N$-gon switches}

The presented multi-layer architectures offer novel all-optical switching fabrics. However, setting up multi-layer architectures may be difficult. Thus their planar realization is desired which is briefly shown in the following by means of the $N$-gon prism switch. Caused by assumptions (A) and (B), for $N$ inputs/outputs, the number of waveguides is $N+1$ (Example 6). However, the doubled waveguide may be chosen arbitrarely out of the $N$ waveguides (Fig. 8) and a path selection switch has to be located at the end of the parallel waveguides (Figs. 9 and 10).

Example 6: For $N=3$ the sitation is obvious whereas for $N=4$ we may switch between the waveguides 1 and 2,1 and 3,2 and 4 and 3 and 4 (spatial diagonal switching between 1 and 4 and 2 
and 3 is not allowed).

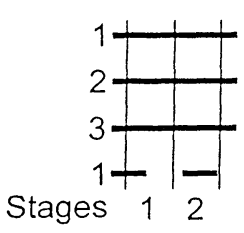

(a)

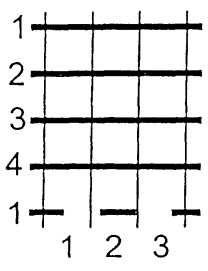

(b)

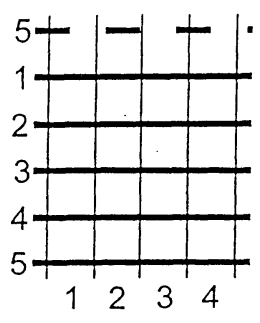

(c)

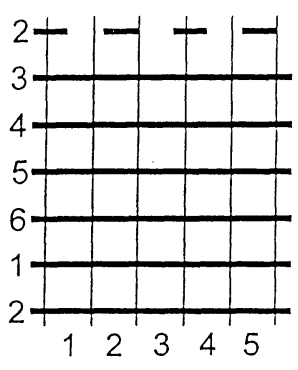

(d)

Fig. 8. Schemes for the planar realization of $N$-gon prism switches for $3 \leq N \leq 6$ (solid lines are common waveguides, dashed lines are additional waveguides where the latter may cause crossings at the inputs and outputs). In principle, any of the $N$ waveguides may be doubled which is verified in Fig. 8 (c) and (d).
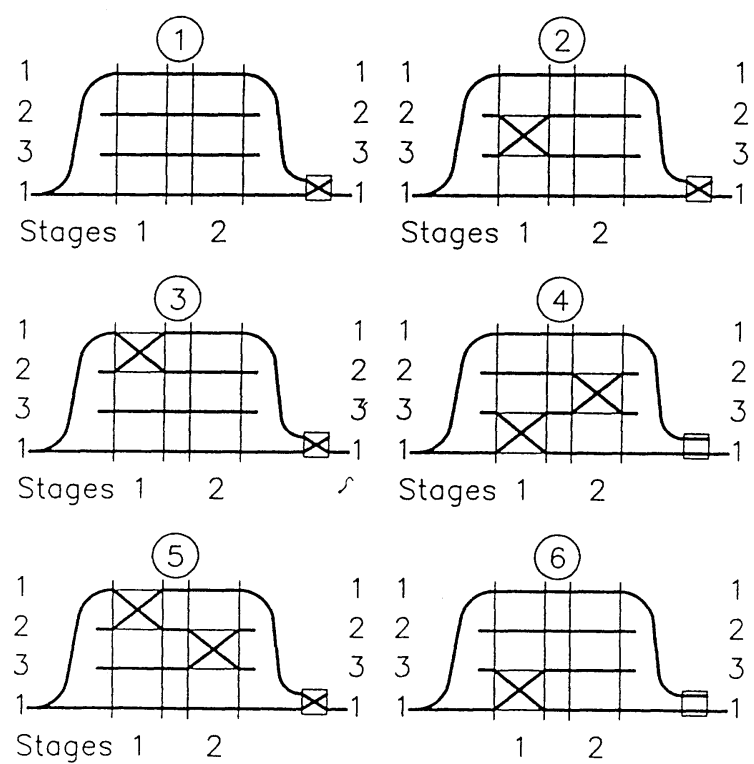

Fig. 9. The $3 !=6$ permutations of the planar 3 -gon prism switch. Note without the doubled waveguide, $N S=3$ for the rearrangeable nonblocking case which equals the Spanke-Benes network result [16]. 
Assumption (A), which is implicitely satisfied in the multi-layer architecture, has to be taken into account by the switch setting algorithm. For example, for $N=3$, if a switch is established between waveguide 1 and 2, within the same stage, no further switch may set up. In contrast, assumption (B), is considered by the number of waveguides and their particular arrangement.

Remark 7: From a comparison of planar $N$-gon prism switches (Figs. 8 to 10 ) with the SpankeBenes network [16] we may conclude a saving of 1 stage $(N S=N-1$ instead of $N)$ and less switches for an odd $N$ according to Eq.(4). However, the prize is an additional waveguide and a path selection switch.

Example 7: For the planar 6-gon prism switch with 5 stages, the number of 15 switches is established by means of 7 parallel waveguides (Fig. 8) and one path selection switch (Figs. 9 and 10) whereas for the embedded 3-gon ${ }^{2}$ prism switch ( 6 inputs/outputs) we have 4 stages, the number of 12 parallel waveguides and and an additional stage with the number of six $2 \times 2$-path selection switches where crossings arise.

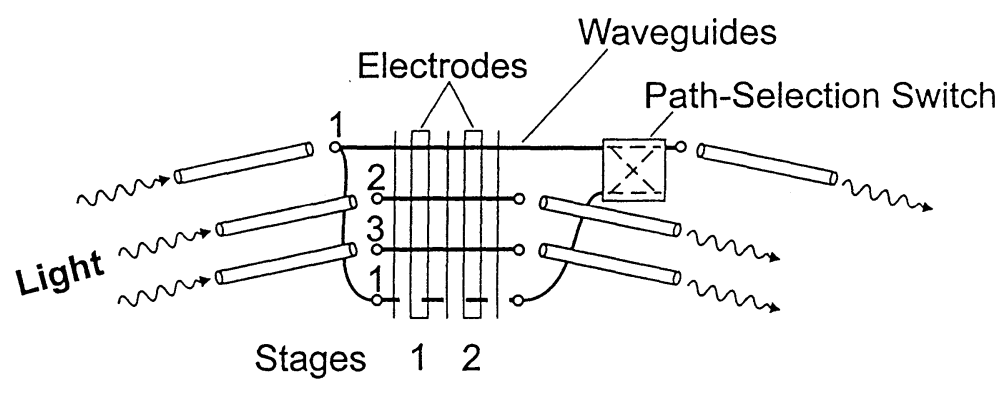

Fig. 10. Scheme of a planar 3-gon prism switch (2 stages, 1 switch/stage, 4 waveguides and 1 path selection switch) instead of the 3 stages and 3 switches of the Spanke-Benes networks [16].

Remark 8: All the proposed multi-layer switching architectures may be realized, in principle, in a planar manner (Sub-Section 3.3) where trade-offs, arise between the parallelism (of waveguides) and $N S$ and the number of crossings.

\subsection{All-optical $O(1)$-switches}

The increase of the number of inputs/outputs of $N$-gon prism switches demands (1) an increase of the number of layers which, by means of the current technology, may be difficult to set up and (2) causes a large $N S$ and in turn a high attenuation of signals. Therefore, the (coset) decomposition of the $N$ ! permutations by means of several $m$-permuters ( $m$-gon prism switches, $m \leq N$ ), as a starting point [26], is a practical solution once the number of permuters is reduced to the minimum [19].

Remark 9: Note the decrease of the number of permuters increases the complexity of the routing in the shells. Beside the reduction of the parallelism, a further problem is the organization of the shells at the lhs and rhs of the parallel $m$-permuters. Three attempts, different also from the point of view of technology, are possible: (a) free-space interconnections (b) cellular arrays (c) expanders and concentrators (Sub-Section 3.5).

Remark 10: By means of (a), the $O(1)$-switch arises in straight-forward way. However, (b) and (c) 
cause additional stages for the expander and concentrator and thus compete with the $N$-gon prism switch.

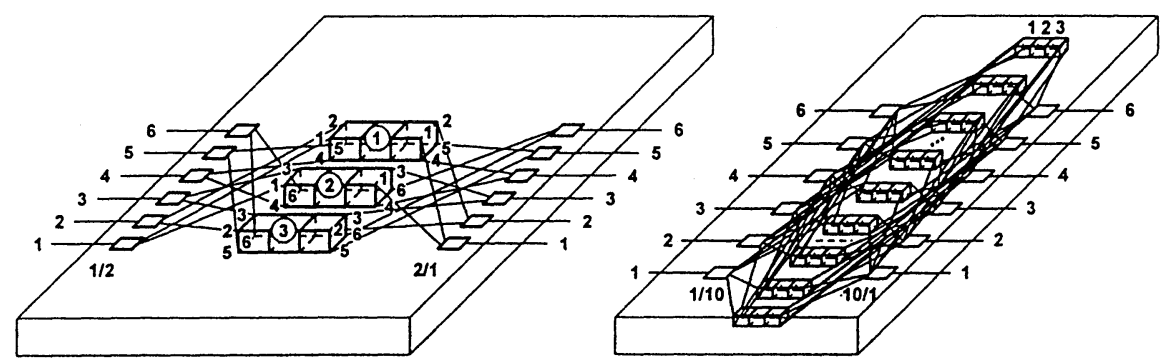

(a)

(b)

Fig. 11. All-optical $O(1)$-switches for 6 inputs/outputs. (a) Rearrangeable nonblocking interconnections [19]. (b) Circuit switching mode [17].

\subsection{Expanders and concentrators}

The distance-transitive graphs [13] may also be applied for the design of all-optical expanders and concentrators. In this way we may organize the shells at the lhs and rhs of the parallel stacked permuters according to the solution (c) (Sub-Section 3.4).
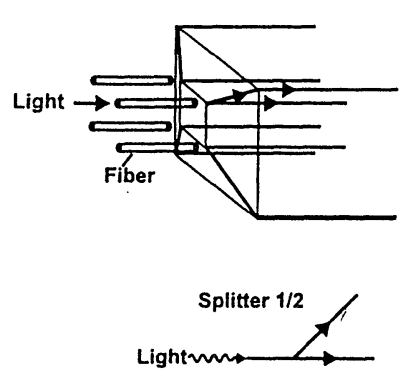

(a)

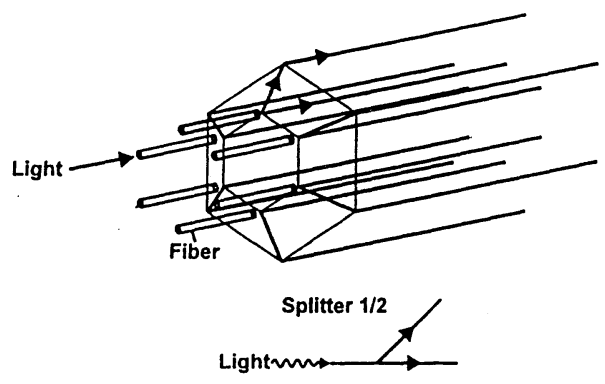

(b)

Fig. 12. Scheme of all-optical expanders (a) $4 / 8$ and (b) 6/12 at the input of an all-optical switch (the reversal is the concentrator at the output of a switch).

The splitting principle of a simple expander is shown in Fig. 12. There the glas fibers carry the light/data to the waveguides which are splitted 1:2 (1:2 also for the $6 \times 6$-switch in Fig. 11, however, 1:3 for an $8 \times 8$-switch) and which forward the light to the three 4-permuters [Fig. 11 (a)]. The 
outputs of the three 4-permuters [Fig. 11 (a)] are combined in the concentrator (Fig. 13) which finally forward the light/data to the output of the switch.

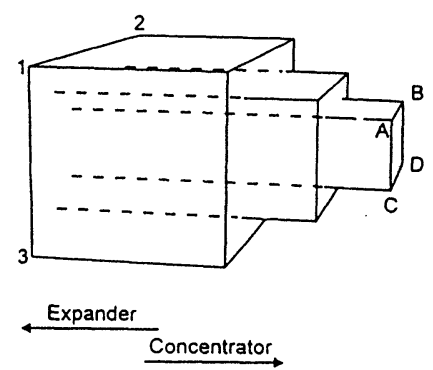

(a)

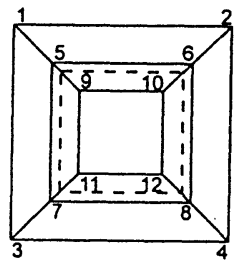

(b)

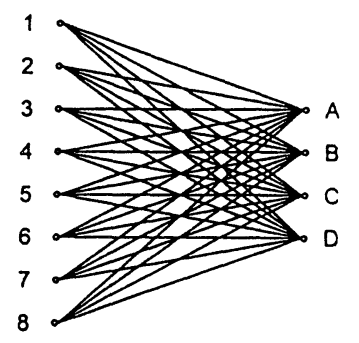

(c)

Fig. 13. (a) Scheme of an expander and concentrator. (b) Intersection. (c) Bipartite graph representing the area between the largest (outer) square and the dashed lined square.

Remark 11: All-optical expanders/concentrators may also be realized in a planar manner (Figs. 8 to 10 ).

\section{Design principles}

Large optical switches usually are designed by the substitution of networks of smaller switches. However, in this way, $N S$ and in turn the attenuation of signals passing through the network increases considerably. Therefore attempts are desired which allow to leave this substitution principle and arrive at proper operating all-optical switches with a large number of inputs/outputs and small $N S$. Once we apply multi-layer architectures with parallel waveguides and electrodes, the following objectives are of interest

- Number of layers

- Number of stages

- Implementation issues.

where the number of layers and $N S$ are dependent, i.e. if the number of layers decreases, $N S$ increases (Table 3). Additionally, the range of possible architectures is very much restricted by the implementation (e.g. switching between embedded $N$-gon prisms by means of electrodes at the outer and inner prism and no new electrode). First of all, we have to chooce the technology/concept (a) to (c) (Sub-Section 3.5) and route signals from the inputs to the permuters and from them to the outputs. Throughout the paper, we discussed expanders and concentrators based on multilayerarchitectures according to (c). However, cases (a) and (b) may also be applied. Note, the planar realization of the switches resolves the implementation difficulties.

There exist construction principles of expanders and concentrators where double covers and the second large eigenvalue comes in $[27,28]$. However, the crucial problem is their all-optical implementation rather than to obtain a large expansion/concentration coefficient and small number of edges. Once we have constructed efficient expanders and concentrators in this way, we may not be able to implement them optically in a spatial manner, except by the resolution of the 
interconnections via several stages. But in this case, we leave the $O(1)$-switch principle, i.e. $N S$ will depend on the number of inputs/outputs.

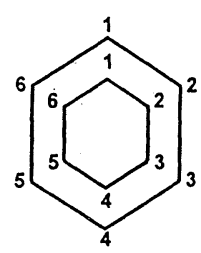

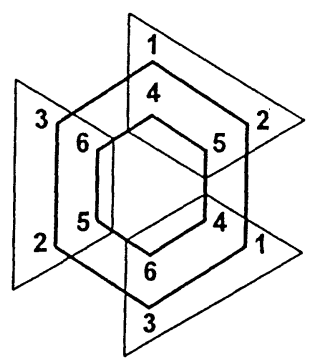

(a)

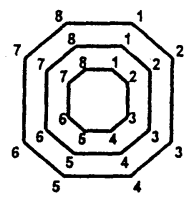

(b)

Fig. 14. Organization of the shells of the proposed optical switch. Intersection of the expanders/concentrators at the lhs and numbering of the parallel permuters/destination routing at the rhs. (a) $6 \times 6$-switch. (b) $8 \times 8$-switch where the inner 4 -permuters switch between 2 and 4,2 and 6 , 4 and 8,6 and 8 (upper switch 2, 4,6 and 8 ) and between 3 and 4,3 and 7,4 and 8 and 7 and 8 (lower switch $3,4,7$ and 8 ).

These problems are solvable by graph topology (coverings, liftings), group theory and combinatorics. For example, Fig. 14 shows, in principle, a covering solution though it was obtained by coset decompositions of permutations (and, in the limit, by the decomposition into 2-cycles) [19]. There exist several nonisomorphic coverings, some of them are countable $[29,30]$, though, in general, their computation is NP-complete [31]. Additionally, we may wish to increase the number of connections between the embedded prisms [32,33], in order to further reduce $N S$ or implement expanders/concentrators. Such an attempts is similar to the multidimensional case in [22], though the implementation of the required $2 \times 2$-switches may be difficult.

\section{Conclusions}

The extension of common planar all-optical switching principles into the 3-D physical space provides novel all-optical switching architectures. The advantages are the least number of $2 \times 2$ switches and the least number of stages for rearrangeable nonblocking interconnections and for the capability to operate in the circuit switching mode. Additionally, the dynamics of the switches (difference of path lengths) is expected to be a minimum. With an extension of the architecture to the embedding of several prism switches and the increase of the connectivity between the prisms, the number of stages may be further reduced. The same principle may be applied to construct all-optical expanders and concentrators. These components may also be applied to implement optically the shells at the lhs and rhs of the parallel permuters which route data from the inputs to the parallel permuters and from the permuters to the outputs. However, the spatial optical implementation may cause considerable difficulties. Therefore, the equivalent planar counterparts have been presented for the simplest case though more complicated architectures may be designed in a planar way according to the principles in Sub-Section 3.3. 


\section{Acknowledgement}

The work is supported by the 'Bundesminister für Bildung, Wissenschaft, Forschung und Technologie' and the City of Berlin within the national PHOTONIK II program under contract 01 BP $449 / 2$.

\section{References}

1. A. Ajmone Marsan, A. Bianco, E. Leonardi and F. Neri, 'De Bruijn topologies for self-routing all-optical networks,' SPIE, vol. 2024, pp.99-111, 1993.

2. C. Berge, Graphs and Hypergraphs, North-Holland Publishing Company, 1973.

3. S. Jiang and T. E. Stern, 'Regular multicast multihop lightwave networks,' INFOCOM'95, paper 6a3, pp.692-700, 1995.

4. W. S. Massey, Algebraic Topology: An Introduction, Springer, New York, 1977.

5. J. L. Gross and Th. W. Tucker, Topological Graph Theory, J. Wiley, New York, 1987.

6. F. Harary, J. P. Hayes and H.-J. Wu, 'A survey of the theory of hypercube graphs,' Comput. Math. Appl., vol. 15, no. 4, pp.277-289, 1988.

7. D. A. Waller, 'Double covers of graphs,' Bull. Austral. Math. Soc., vol. 14, pp.233-248, 1976.

8. D. Angluin and A. Gardiner, 'Finite common coverings of pairs of regular graphs,' J. Combin. Theory, vol. B-30, pp.184-187, 1981.

9. J. R. Munkres, Topology, a 1st course, Prentic Hall, Englewood Cliffs, 1975.

10. W. Kautz, K. N. Levitt and A. Waksman, 'Cellular interconnection arrays,' IEEE Trans. Comput., vol. C-17, no. 5, pp.162-170, May 1968.

11. HHI, Optical Switch Routing Subroutine Library, 1996.

12. D. Z. Djokovic, 'Automorphism of graphs and coverings,' J. Combin. Theory, vol. B-16, pp.243-247, 1974 .

13. N. Biggs, Algebraic Graph Theory, Cambridge University Press, 1974.

14. R. M. Tanner, 'Explicit concentrators from generalized $N$-gons,' Siam J. Alg. Disc. Meth., vol. 7 , no. 3, pp.287-293, September 1984.

15. M. Farzan and D. A. Waller, 'Antipodal embeddings of graphs,' J. London Math. Soc., vol. 15-2, pp.377-383, 1977.

16. R. A. Spanke and V. E. Benes, ' $N$-stage planar optical permutation network,' Appl. Opt., vol. 26, no. 7, pp.1226-1229, 1987.

17. J. Giglmayr, 'Novel all-optical planar and compact minimum-stage switches of size $\geq 4 \times 4$,' SPIE 2918, pp.240-258, 1996.

18. J. Giglmayr, 'Principles of all-optical switching fabrics with minimum number of stages,' Spring Topical Meeting Optics in Computing, Hyatt Lake Tahoe, Incline Village, Nevada, USA, 17-21 March, 1997. 
19. J. Giglmayr, 'Reduction of parallelism in optical $O(1)$-switches,' Conference on Information Sciences and Systems, Johns Hopkins University, Baltimore, 19-21 March, 1997.

20. T. Pisanski, J. Shawe-Taylor and J. Vrabec, 'Edge-colorability of graph bundles,' J. Combin. Theory, vol. B-35, pp.12-19, 1983.

21. A. Graham, Kronecker Products and Matrix Calculus with Applications, E. Horwood, 1981.

22. J. Giglmayr, 'Rearrangeability and connectivity of multistage interconnection networks with nearest-neighbour interconnections,' IEICE Trans. Commun. (Japan), vol. E77-B, no.12, pp.1546-1555, December 1994.

23. A. Blass, F. Harary and Z. Miller, 'Which trees are link graphs,' J. Combin. Theory, vol. B-29, pp.277-292, 1980.

24. J. Giglmayr, 'Kautz topologies for all-optical self-routing networks,' SPIE 2614, pp.168-182, 1995.

25. G. E. Carlson, J. E. Cruthirds, H. B. Sexton and C. G. Wright, 'Interconnection networks based on a generalization of cube-connected cycles,' IEEE Trans. Comput., vol. C-34, no. 8, pp.769-772, August 1985.

26. A. Y. Oruc, 'Designing cellular permutation networks through coset decompositions of symmetric groups,' J. Parallel and Distributed Computing, vol. 4, pp.404-422, 1987.

27. G. A. Margulis, 'Explicit constructions of concentrators,' Problemy Peredachi Informatsii, vol. 9, no. 4, pp.71-80, 1973.

28. O. Gabber and Z. Galil, 'Explicit constructions of linear-sized superconcentrators,' J. Computer and System Science, vol. 22, pp.407-422, 1981.

29. M. Hofmeister, 'Counting double covers of graphs,' J. Graph Theory, vol. 12, no. 3, pp.437-444, 1988.

30. S. Hong and J. H. Kwak, 'Regular fourfold coverings with respect to the identity automorphism,' J. Graph Theory, vol. 17, no. 5, pp.621-627, 1993.

31. VEGA, Graph Gallery, version 0.2, University of Ljubljana, 1995.

32. M. Farzan and D. A. Waller, 'Kronecker products and local joins of graphs,' Can. J. Math., vol. 24 , no. 2 , pp.255-269, 1977.

33. N. Hartsfield and G. Ringel, Pearls in Graph Theory, Academic Press, 1994. 